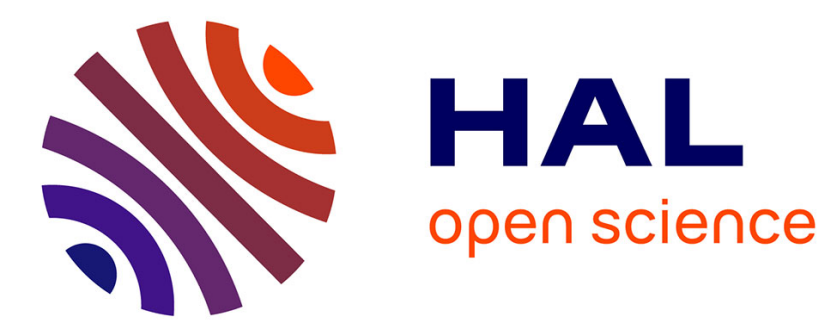

\title{
Protection des transistors MOS en régime de deuxième claquage
}

\author{
Henri Tranduc, P. Rossel
}

\section{To cite this version:}

Henri Tranduc, P. Rossel. Protection des transistors MOS en régime de deuxième claquage. Revue de Physique Appliquée, 1982, 17 (6), pp.389-391. 10.1051/rphysap:01982001706038900 . jpa-00245011

\section{HAL Id: jpa-00245011 https://hal.science/jpa-00245011}

Submitted on 1 Jan 1982

HAL is a multi-disciplinary open access archive for the deposit and dissemination of scientific research documents, whether they are published or not. The documents may come from teaching and research institutions in France or abroad, or from public or private research centers.
L'archive ouverte pluridisciplinaire HAL, est destinée au dépôt et à la diffusion de documents scientifiques de niveau recherche, publiés ou non, émanant des établissements d'enseignement et de recherche français ou étrangers, des laboratoires publics ou privés. 
Classification

Physics Abstracts

$65.00-72.80$

\title{
Protection des transistors MOS en régime de deuxième claquage
}

\author{
H. Tranduc et P. Rossel \\ Laboratoire d'Automatique et d'Analyse des Systèmes du C.N.R.S., \\ 7, avenue du Colonel Roche, 31400 Toulouse, France
}

(Reçu le 25 novembre 1981, révisé le 4 mars 1982, accepté le 10 mars 1982)

\begin{abstract}
Résumé. - On propose une méthode originale de stabilisation du fonctionnement des transistors MOS dans le régime de deuxième claquage. Elle est basée sur l'utilisation d'une résistance intégrée et répartie de source, dite " ballast ", qui, par contre-réaction électrique, évite les mécanismes de concentration et de focalisation des lignes de courant et protège le composant contre toute destruction par point chaud localisé.
\end{abstract}

\begin{abstract}
A new method for stabilizing the operating point of MOS transistors in the second breakdown state, is proposed : the current crowding mechanism is avoided by using the electrical feed back of a distributed ballast resistance integrated in series with the source electrode.
\end{abstract}

\section{Introduction : définition de l'état de deuxième} claquage. - Lorsqu'on augmente la tension drainsubstrat d'un transistor Métal-Oxyde-Semiconducteur, la jonction source-substrat étant court-circuitée, le champ électrique qui règne dans la zone de pincement de canal, atteint des valeurs telles qu'il se produit au sein de celle-ci une ionisation par choc, création de paires électron-trou, et multiplication du courant [1]. Le transistor fonctionne en régime dit d'avalanche. $\mathrm{Si}$ on continue à faire croître le courant drain, il apparaît, à partir d'un certain niveau, un second état présentant d'abord une résistance négative, puis un régime à haut niveau de conduction en courant pour une faible tension de maintien. Ce dernier état qui a été mis en évidence pour la première fois par Asakawa et al. [2], est appelé l'état de deuxième claquage. Ce régime est difficile à observer dans des conditions statiques car le transistor se détruit alors quasiinstantanément. En régime impulsionnel, la mise en évidence du lieu de second claquage est possible, et la figure 1 est un exemple de tracé des caractéristiques de sortie relevé par la méthode de Nakagiri [3] sur un transistor MOS plan à structure classique.

2. Le phénomène et les protections actuelles. Ce phénomène de deuxième claquage est associé $[4,5]$ à un mécanisme de réaction électrique-thermique en plusieurs étapes (Fig. 2) :

1) Une ionisation par choc due aux porteurs du courant de canal dans la zone pincée près du drain entraîne la création de paires électron-trou;

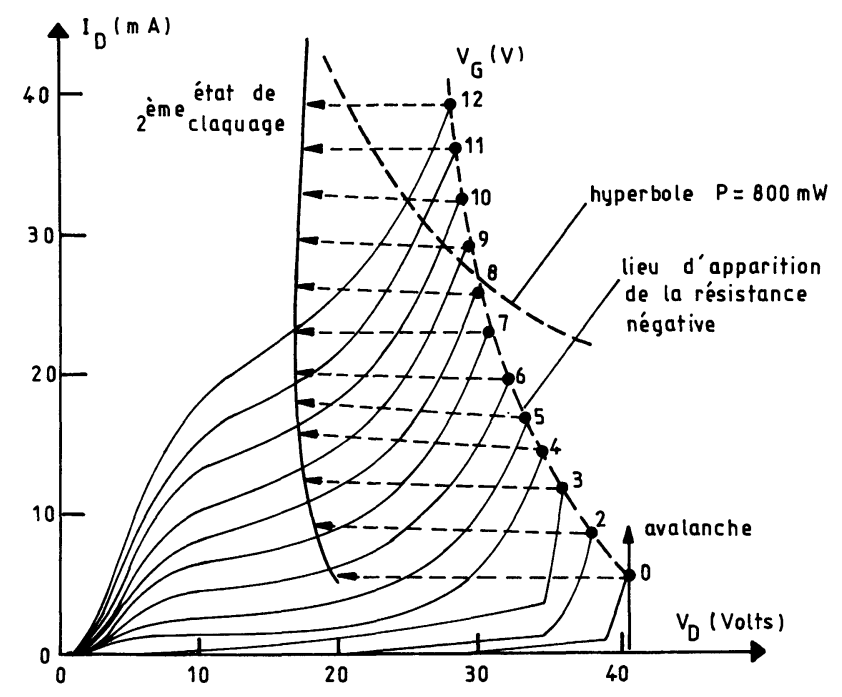

Fig. 1. - Caractéristiques de sortie d'un transistor MOS montrant les régimes de fonctionnement normal, d'avalanche et de deuxième claquage. Mode pulsé (rampe de courant de $2,5 \mu$ s de durée). - T. MOS Canal $N$ - Structure $\mathrm{N}^{+} \mathrm{PN}^{+}-L \simeq 6 \mu \mathrm{m}-$ dopage de substrat $\simeq 10^{16} \mathrm{~cm}^{-3}$.

[Experimental MOS transistor output characteristics showing the three operating modes : normal, avalanche breakdown, second breakdown. $2.5 \mu$ s wide pulses at a repetition rate of $100 \mu \mathrm{s}$. N-channel $\mathrm{N}^{+} \mathrm{PN}^{+}$MOS'T; channel length $L \simeq 6 \mu \mathrm{m}$, substrate doping value $\simeq 10^{16} \mathrm{~cm}^{-3}$.]

2) L'évacuation des trous se fait vers le contact du substrat; 


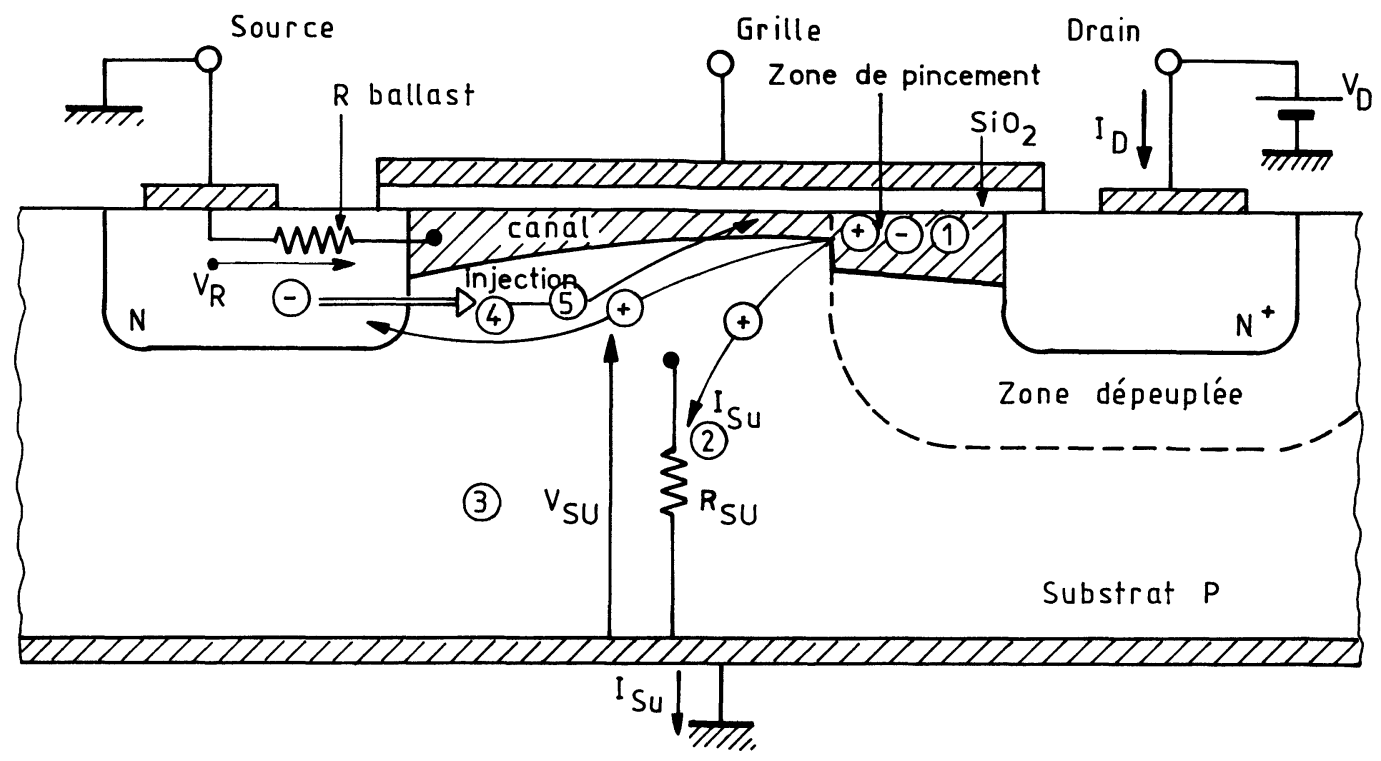

Fig. 2. - Coupe d'un transistor MOS avec schématisation des phases du mécanisme de second claquage ainsi que de la localisation de la zone « ballast » de source.

[Cross-section of a N-channel MOS transistor showing the mechanisms involved in the second breakdown effect and indicating the source ballast resistance location.]

3) Ce courant de trous développe dans la résistance du substrat, une chute de potentiel qui polarise la source négativement par rapport au substrat;

4) Le substrat au voisinage de la source étant positif, la diode source-substrat devient passante : les trous sont drainés vers la source et corrélativement de nouveaux électrons sont injectés vers le drain via le substrat;

5) Ces porteurs contribuent à leur tour à la multiplication par avalanche au droit du drain et la boucle d'instabilité électrique est ainsi fermée.

6) Il se produit alors une augmentation localisée de puissance et de température, et l'apparition d'une zone conductive très chaude liée à des effets focalisants (i) transversal - sous l'action du champ électrique grille-substrat - et (ii) longitudinal [2] - conduction filamentaire initiée par les inhomogénéités, dislocations ou surdosage et imperfection du substrat près du drain [7] et accrue par l'instabilité vis-à-vis du comportement thermique de la boucle 1-6.

Ces effets focalisants induisent une situation d'échauffement cumulatif local pouvant aller jusqu'à la mise en court-circuit et la fusion de la jonction drain-substrat. Cet effet est la cause principale de défaillance des transistors MOS multicellulaires ou des structures MOS de puissance.

Jusqu'à ce jour, dans le but de protéger les transistors MOS contre la destruction en second claquage, diverses configurations géométriques ou technologiques ont été proposées et sont effectivement utilisées. Elles sont basées sur les trois principes suivants : (i) diminution de la valeur de la résistance de substrat, (ii) éloignement de la zone d'avalanche par rapport à la source par utilisation d'une grille écran [5], (iii) élimination de l'avalanche de canal en shuntant le composant par une diode N-P intégrée à tension de claquage plus faible que celle du T. MOS [6].

Ces méthodes sont des artifices qui, de fait, empêchent le réseau de caractéristiques de la partie active du transistor MOS, d'atteindre le. lieu où la résistance négative, et par suite, le basculement en $2^{\mathrm{e}}$ claquage se produisent. La conséquence principale en est une limitation forcée en valeur de tension d'utilisation du dispositif par rapport à ce que l'on pourrait obtenir intrinsèquement.

3. Utilisation d'une résistance répartie dans la source. - Nous proposons, pour notre part, une méthode de stabilisation de la caractéristique couranttension en régime de deuxième claquage, contre les effets focalisants et l'apparition de point chaud. Elle consiste à intégrer lors de la fabrication du transistor MOS une résistance d'accès, de nature répartie, en série avec la source du transistor. Le rôle de cette résistance est d'introduire un effet de contre-réaction électrique, se manifestant par une apparition locale de tension source-substrat, $V_{\mathbf{R}}$ (Fig. 2), variable suivant les effets de concentration des lignes de courant. Cette tension polarise en inverse la jonction source-substrat et fait diminuer localement aussi bien le courant du canal d'inversion que le courant dû à l'injection d'électrons de la source vers le substrat. Le transistor est ainsi stabilisé et peut fonctionner sur son lieu de deuxième claquage. La figure 3 représente le type de résultats expérimentaux relevés sur un traceur ordinaire de caractéristiques et obtenus à partir de transistors MOS à canal $\mathrm{N}$ dont la source 


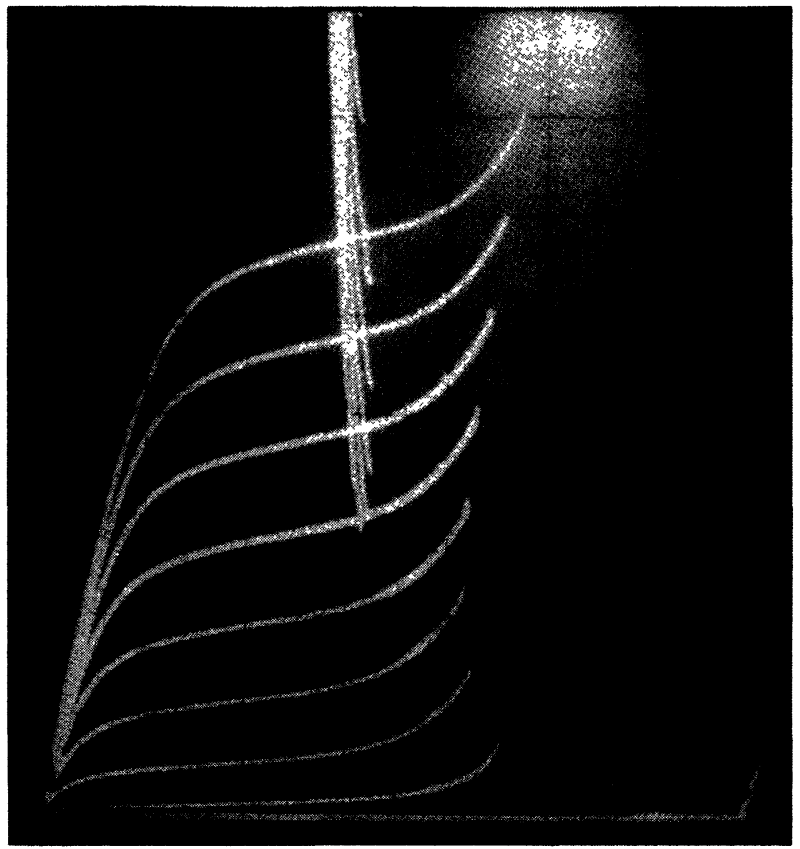

Fig. 3. - Caractéristiques de sortie d'un transistor MOS " protégé " contre les instabilités en deuxième claquage. Mise en évidence du fonctionnement stable dans ce régime. - Mode $50 \mathrm{~Hz}$ (traceur Tektronix - type 577-177-D1) vert. $1 \mathrm{~mA} /$ div. - horiz. $2 \mathrm{~V} /$ div. - saut de $V_{G}=1 \mathrm{~V}$ $V_{\mathrm{G}} \max =10 \mathrm{~V}$. $\bullet \mathrm{T}$. MOS - canal N-Structure $\mathrm{NPN}^{+}$ $-L<2 \mu \mathrm{m}-$ dopage de substrat $\simeq 5 \times 10^{16} \mathrm{~cm}^{-3}$.

[Experimental output characteristics of a MOS transistor protected against second breakdown induced failures. $50 \mathrm{~Hz}$ - Tektronix 577-177-D1 curve tracer. Horiz. $2 \mathrm{~V} /$ div., Vert. $1 \mathrm{~mA} /$ div., 10 gate steps of $1 \mathrm{~V}$. N-channel $\mathrm{NPN}^{+}$, channel length $L<2 \mu \mathrm{m}$, substrate doping value $\simeq 5 \times 10^{16} \mathrm{~cm}^{-3}$.]

est « ballastée " par résistance répartie. Ces composants ont une longueur de canal inférieure à $2 \mu \mathrm{m}$ et une largeur $Z$ de $100 \mu \mathrm{m}$; le substrat est dopé à $5 \times 10^{16}$ impuretés par $\mathrm{cm}^{3}$, et la résistance ballast est constituée par une zone d'accès de source de $5 \mu \mathrm{m}$ de long sur $10 \mu \mathrm{m}$ environ d'épaisseur, dopée de type $\mathbf{N}$ à $2 \times 10^{15}$ impuretés par $\mathrm{cm}^{3}$. La résistance correspondante est d'environ $1 \Omega$ par centimètre de largeur du transistor. Elle représente le quart de la valeur limite de la résistance à l'état passant, $R_{\mathrm{ch}}$, du canal actif qui est explicitée par [8] :

$$
R_{\mathrm{ch}}=\left(\mu_{0} \cdot \frac{Z}{L} \cdot C_{\mathrm{ox}} \cdot \psi\right)^{-1}
$$

où $\mu_{0}$ est la mobilité dans la couche inversée à faible champ électrique, $\psi$ le potentiel de réduction transversale de mobilité et $C_{\text {ox }}$ la capacité d'oxyde de grille par unité de surface.

On a pu relever sur ces structures protégées que le fonctionnement sur le lieu de deuxième claquage est permis en régime continu sans qu'une défaillance catastrophique se manifeste.

4. Conclusion - On a montré expérimentalement que la présence d'une résistance répartie de source permet la stabilisation du fonctionnement électrique du transistor MOS en régime dit de deuxième claquage. Cette méthode déjà connue pour le transistor bipolaire [9] est originale dans le cadre du MOST et son principal intérêt par rapport aux autres méthodes proposées est de ne pas limiter artificiellement la tension maximale applicable au drain.

Son inconvénient majeur est d'augmenter la résistance à l'état passant du transistor. Compte tenu du fait qu'il semble qu'un ordre de grandeur convenable pour cette résistance soit du quart de la résistance du canal actif, cet inconvénient est d'autant moins critique que la tension d'avalanche est haute. En particulier, dans le cas de transistors MOS de puissance de moyenne ou haute tension, cet inconvénient disparaît complètement puisque la résistance à l'état passant n'est imposée [8] que par la résistance de la couche peu dopée de drain qui a une valeur plus grande que celle de la résistance du canal ou celle de la résistance « ballast » de source nécessaire.

\section{Bibliographie}

[1] Martinot, H., Rossel, P., Multiplication des porteurs dans la zone de pincement des transistors MOS, Electron. Lett. 7 (1971) 118.

[2] Asakawa, T., Tsubouchi, N., Second Breakdown in MOS Transistors, IEEE Trans. Electron Devices ED-13 (1966) 811.

[3] NAKagiRI, M. and IIDA, K., Damage introduced by Second Breakdown in N-channel MOS devices, Japan. J. Appl. Phys. 16 (1977) 1187.

[4] Toyabe, T., Yamaguchi, K., Asai, S. and Mock, M. S., A Numerical Model of Avalanche Breakdown in MOS FET's, IEEE Trans. Electron Devices ED-25 (1978) 825.

[5] Yoshida, I., OKabe, T., Katsueda, M., OChi, S. and NaGata, M., Thermal Stability and Secondary Breakdown in Planar Power MOSFET's, IEEE Trans. Electron Devices ED-27 (1980) 395.
[6] Tihanyi, J., A Qualitative Study of the DC Performance of SIP MOS Transistors Siemens Forsch. (Entwickl) Ber. Bd. 9 (1980) 181.

[7] Smith, W. B., Pontius, D. H. and Budenstein, P. P., Second breakdown and damage in junction devices, IEEE Trans. Electron Devices ED-20 (1973) 731.

[8] Rossel, P., Tranduc, H., Gamboa, M., Phan Pham, T., Limitation Fondamentale dans les T. MOS de puissance : le compromis entre la résistance à l'état passant $R_{\mathrm{ON}}$ et la tension' de claquage $V_{\mathrm{DBR}}$, Revue Phys. Appl. 16 (1981) 509

[9] RosenzWeig, R. and CARLEY, D. R., Design of a VHF power transistor with second breakdown protection, IEEE International Electron Devices Meeting, Washington D.C., October 1965. 